\title{
Mehrsprachigkeit in der Bildung in Deutschland - eine Diskursanalyse
}

\author{
FARIN ENGELS
}

\begin{abstract}
Multilingualism in Education in Germany - a Discourse Analysis. In the Republic of Germany, language acquisition for children with a mother tongue other than German has been a widely discussed topic in education science as well as in public and political discourse over the last decades. Annual studies on preschool and primary education point to the ongoing disadvantage - or even discrimination - suffered by multilingual children in the German education system. Given Germany's history as a country of immigration, and in light of recent public discussions on the increased immigration of refugees, the question of the problematic's socio-political background arises. This leads to the issue of linguistic concepts among society and their influence on domestic language policies. This article presents analysis of discourse around languagepolitical concepts and practices among stakeholders in language promotion in the state of North-Rhine Westphalia. Five semi-structured interviews with state employees were analysed following a discourse analytical approach. The analysis, deploying frameworks from the field of Critical Discourse Analysis, focused on the discursive practice of legitimation in the evaluation of multilingualism. Multilingualism as a phenomenon in society appeared to be evaluated according to differing standards (for example correctness or properness) depending on the language. As for evaluation, the analysis indicated an additional dimension in discourse which assigns different groups of speakers a belonging to particular languages. All in all, the collected data pointed to a diverse and ever-changing discourse in the field of language promotion in North-Rhine Westphalia. The presented analysis aims to stimulate a debate and suggests some directions for future research.
\end{abstract}

Keywords: multilingual education; discourse analysis; Germany; multilingualism; reading literacy 
Mehrsprachigkeit in der Bildung in Deutschland - eine Diskursanalyse

\section{Einleitung}

Die öffentliche und politische Diskussion über den Umgang mit Spracherwerb in der Schule hat in Deutschland, das von jeher Einwanderungsland ist (Bildungsbericht 2016: 161), Tradition. Spätestens seit der Anwerbung ausländischer Gastarbeiter in den 1950er Jahren unterlagen Sprach- und Bildungspolitik in Deutschland ständigen strategischen Richtungswechseln. ${ }^{1}$ Die vermehrte Zuwanderung von Flüchtlingen um das Jahr 2015 hat Debatten um die Ziel- und Umsetzung bildungspolitischer Maßnahmen zur Unterstützung von Integration und, damit verbunden, des Erwerbs der deutschen Sprache, wieder verstärkt in den Fokus gerückt. Neben der intensiven Beschäftigung mit Deutsch als Zweitsprache bzw. Deutsch als Fremdsprache spielt die tatsächliche Sprachvielfalt an deutschen Schulen - und das Potential, die vorhandenen Sprachen in Bildung miteinzubeziehen - allenfalls eine untergeordnete Rolle. Einer der Gründe hierfür scheint ein Mangel an Bewusstsein für die sprachlichen Hintergründe der Schüler sowie für sprachpolitische Themen im Allgemeinen zu sein, wie im Verlauf dieses Texts weiter erläutert werden wird.

Der vorliegende Artikel beschäftigt sich mit der Frage, wie sich Verantwortliche im Bereich der Sprachförderung an Schulen individuelle Mehrsprachigkeit vorstellen und wie diese Konzepte auf diskursiver Ebene widergespiegelt werden.

Die Ergebnisse meiner Analyse von fünf Interviews deuten darauf hin, dass das Prinzip individueller Mehrsprachigkeit bei einigen der befragten Experten für die sprachliche Entwicklung von Schülern hinter einer Wahrnehmung von einsprachigen Kindern zurückblieb. Die Bewertung von Prozessen des Spracherwerbs und der Sprachkompetenz schien für Deutsch und für nichtdeutsche Sprachen unterschiedlichen Kriterien zu unterliegen. Außerdem schien die Idee von mehrsprachiger Bildung in der Schule häufig einem empfundenen Zweck einsprachig deutscher Bildung untergeordnet $\mathrm{zu}$ werden. Insgesamt wies der Gebrauch von diskursiven Praktiken der Legitimation auf Konzeptionen von Mehrsprachigkeit hin, die eher auf moralischen Bewertungen als auf sprachwissenschaftlichen Konzepten beruht.

Um die Ergebnisse und ihre anschließende Diskussion einordnen zu können, muss zunächst der Kontext, in dem Mehrsprachigkeit in der Schule behandelt wird, berücksichtigt werden.

1 Einen Überblick über Bildungspolitik und Mehrsprachigkeit im Verlauf der letzten Jahrzehnte gibt Belke (2016: 12-21). 
ENGELS

Sprachpolitische Praxis und mögliche Diskriminierung in früher Bildung

Die Dringlichkeit und Relevanz einer Bildungspolitik, die die Sprachvielfalt an deutschen Schulen berücksichtigt, wurde spätestens mit der Veröffentlichung der Ergebnisse des Programme for International Student Assessment (PISA) im Jahr 2001 zum Diskussionsgegenstand einer breiteren Öffentlichkeit. Die OECD-Studie bescheinigte dem deutschen Bildungssystem den größten Zusammenhang zwischen sozialer Herkunft von Schülern und ihrer Teilhabe an Bildung unter allen teilnehmenden Ländern (Artelt et al. 2001: 40). Insbesondere der Vergleich von Kindern deutscher Eltern und Kindern, deren Eltern beide nach Deutschland eingewandert waren, zeigte drastische Unterschiede in ihren Chancen auf Bildungserfolg. Die Lesefähigkeiten von $50 \%$ der letztgenannten Gruppe gingen nicht über die erste Stufe von fünf in der Studie erarbeiteten Kompetenzstufen hinaus. Für diese Schüler bezeichneten Stanat et al. in ihrer Analyse der Ergebnisse sprachliche Fähigkeiten als „die entscheidende Hürde in ihrer Bildungskarriere" (2002: 13).

Seit der Veröffentlichung der Studie, die auch als „PISA-Schock“ für die deutsche Gesellschaft bezeichnet wird, haben Studien im Verlauf der letzten 20 Jahre wiederholt auf die Korrelation zwischen Bildungsbenachteiligung und sprachlichen Fähigkeiten mehrsprachiger Kinder hingewiesen (Bildungsbericht 2006: 173, Bildungsbericht 2016: 9, Aydın-Canpolat 2018: 102). Trotz zahlreicher Diskussionen und Neuansätze in der Bildungspolitik scheint die Frage nach einer einheitlichen und effektiven Sprachpolitik, die Schülerinnen und Schülern Bildungsteilhabe unabhängig von ihrer Muttersprache ermöglicht, weiterhin unbeantwortet.

Der Anteil der Kinder, die in Deutschland mit einer anderen Muttersprache als Deutsch aufwachsen, ist nicht genau belegt. Ein Bedarf an repräsentativen Daten zur sprachlichen Realität in Deutschland lässt sich allerdings an einer Häufung von Veröffentlichungen der letzten Jahre ablesen: 2017 nahm das Statistische Bundesamt im Mikrozensus erstmals in dessen Geschichte eine Frage zur im Haushalt der Befragten vorwiegend gesprochenen Sprache auf (Adler 2019: 197). Adler (2019) betont, dass die vom Leibniz-Institut für Deutsche Sprache durchgeführte Deutschland-Erhebung von 2018 (in Druck) zusammen mit dem genannten Mikrozensus die einzige repräsentative Datengrundlage für in Deutschland gesprochene Sprachen darstellt. Dieser Mangel an detaillierten Daten - bzw. ihre für sprachwissenschaftliche Zwecke bedenkliche Erhebung und Interpretation, vgl. Adler (2019: 204) kann als deutlicher Hinweis auf einen allgemeinen Mangel an Bewusstsein 
oder Verständnis für die Vielfalt an in Deutschland gesprochenen Sprachen verstanden werden.

Die Autoren des Bildungsberichts 2016 gaben an, erstmals exemplarische Daten zu den Familiensprachen von Vier- bis Fünfjährigen mit Migrationshintergrund liefern zu können (Bildungsbericht 2016: 166). Deutschlandweit sprachen $63 \%$ der Kinder, denen ein Migrationshintergrund zugeschrieben wird und die eine Tageseinrichtung besuchen, zu Hause eine andere Sprache als Deutsch. Anhand von Elternbefragungen wurde festgestellt, dass bei fast einem Viertel aller Drei- bis Fünfjährigen in Deutschland ein Bedarf für Sprachförderung in Deutsch diagnostiziert wurde. Dabei muss betont werden, dass der Anteil bei Kindern, die in der Familie eine nicht-deutsche Familiensprache sprechen, 39\% betrug, und für Kinder mit Deutsch als Familiensprache 21\% (Bildungsbericht 2016: 66). Im Zusammenhang mit diesen recht hohen Anteilen in beiden Gruppen benennen die Autoren des Bildungsberichts 2016 den Bildungsabschluss der Eltern als wichtigen Faktor.

Insgesamt ist laut dem Bildungsbericht 2016 der Anteil der Menschen mit Migrationshintergrund höher, je jünger die untersuchte Altersgruppe ist. Eine Auswertung von Daten aus dem Jahr 2013 zeigte, dass im nationalen Durchschnitt mehr als ein Drittel der unter Zehnjährigen Eltern oder Großeltern haben, die nach Deutschland zugewandert sind (Bildungsbericht 2016: 165). Im Jahr 2018 (Bildungsbericht 2018: 74) hatten 28\% der Kinder in Kindertagesbetreuung einen Migrationshintergrund. Etwa 65\% von ihnen sprachen zu Hause eine andere Sprache als Deutsch.

Diskussionen um den Umgang mit den bereits vorhandenen nichtdeutschen Muttersprachen von Schülern haben also Auswirkungen auf einen beachtenswerten Teil der Schülerschaft.

Aus soziolinguistischer Perspektive hat sich Nathalie Thomauske (2017) mit Sprachpolitik und -praktiken in deutschen und französischen Vorschuleinrichtungen befasst. In ihrer Dissertation Sprachlos gemacht in Kita und Familie untersucht sie ausführlich Faktoren von Diskriminierung, die zum Verstummen mehrsprachiger Kinder führen kann; dabei legt sie besonderes Augenmerk auf historisch-ideologische Hintergründe für die untersuchten Praktiken.

Insgesamt bescheinigt Thomauske den beiden untersuchten Bildungskontexten eine hohe Anpassungserwartung gegenüber Kindern mit nichtdeutscher Muttersprache (2017: 241). Wo Merkmale mehrsprachiger Lern- und Kommunikationsprozesse (zum Beispiel die Mischung mehrerer Sprachen) auftreten, entsprechen diese möglicherweise nicht der Erwartung an eine einsprachige Person. In diesem Zusammenhang beschreibt Thomauske eine 
ENGELS

„Tradition des sprachlichen Handicaps“ (2017: 316). ${ }^{2}$ Hier wird ein Kontext beschrieben, in dem Phänomene mehrsprachigen Spracherwerbs missverstanden und in der Folge problematisiert werden. Gleichzeitig weist Thomauske (2017: 319) auf Diskussionen um Bildungspolitik der letzten Jahrzehnte hin, in deren Verlauf getroffene bildungspolitische Entscheidungen den erfolgreichen Erwerb mehrerer Sprachen in vielen deutschen Schulen bisher behindert haben. Besonders die Segregation von Kindern in verschiedene Formen gesonderter Klassen oder Sprachfördermaßnahmen und eine damit einhergehende Wertung der Kompetenzen mehrsprachiger Kinder als defizitär gegenüber derer einsprachig deutsch aufwachsender Kinder beschreibt sie als Mechanismus, der eine Mentalität der Normsprachigkeit und Anderssprachigkeit befördert.

Hinsichtlich der Hierarchisierung von Sprachen in deutschen und französischen Bildungseinrichtungen bezieht sich Thomauske (2017) ausführlich auf die Ideologie und Geschichte der Nationalstaatenbildung in beiden Ländern. Zum Zusammenhang zwischen Nationalstaat und Sprache schreibt sie:

Die Vorstellung einer Nation, in der davon ausgegangen wird, dass eine Zugehörigkeit zur Nation nur vererbt werden könne und jemand nur dann „richtig“ deutsch wäre, wenn die „Muttersprache“ und die Vorfahren deutsch wären, hat bis heute weitreichende Konsequenzen [...] (Thomauske 2017: 60)

Als eine dieser Konsequenzen beschreibt Thomauske eine eindeutige Hierarchie von Sprachen, in der nicht-deutschen Muttersprachen von Kindern ein niedrigerer Status als der dominanten Bildungssprache Deutsch zugewiesen wird (2011: 332). Aus einem solchen Verständnis von Nationalsprache, und ihrer Instrumentalisierung auf politisch-administrativer Ebene (basierend auf dem Argument „deutscher Leitkultur"), resultieren laut Thomauske (2017: 210ff) bis heute Praktiken in Bildungseinrichtungen, die mehrsprachige Kinder bei Bestehen auf dem Gebrauch der Nationalsprache benachteiligen oder ausschließen. Wo Kindern die Gelegenheit fehlt, ihre bereits erworbene Sprache zu sprechen, oder wo der Gebrauch einer nicht-deutschen Sprache verboten oder

2 Thomauske (2017: 320) beschreibt näher: „Die Vorstellung, dass Sprachmischung eine Unzulänglichkeit sei, die eine Halbsprachigkeit oder Semilingualismus symbolisiere bzw. dazu führe und demnach eine Inkompetenz Mehrsprachiger darstelle, die ihre Sprachen nicht fein säuberlich trennen würden, erinnert an die Sprachideologie des 16. Jahrhunderts: des ,Sprachreinheitsgebots', die sich als Sprachnorm während der Nationenbildung und dem Kolonialismus entwickelte." 
abgewertet wird, kann dies zu Rückzug und Verstummen der Kinder führen (vgl. Thomauske 2017: Kap 5.2.2).

Zur Sprachförderung in Deutschland merkt Thomauske (2017: 12) an, dass ein Großteil der Maßnahmen nicht auf sprachwissenschaftlicher Erkenntnis basiert. Des Weiteren betont Thomauske (2011: 335), dass in der Bildung Tätige oft unzureichend für den Umgang mit mehrsprachigen Kindern ausgebildet sind, und dass sie sich sprachwissenschaftlicher Erkenntnisse nicht bewusst zu sein oder sie zu ignorieren scheinen.

Die Effekte von mangelndem Fachwissen im Bereich der Mehrsprachigkeit auf junge Lernende hat auch Gönül Aydın-Canpolat (2018) untersucht und in ihrer umfassenden Studie über den Zusammenhang zwischen dem Migrationshintergrund von Lernenden und ihrem Bildungserfolg wiederholt auf den Faktor der Sprache hingewiesen. In ihrer Dissertation Erfolg und Scheitern im Deutschen Bildungswesen. Determinanten der Bildungsverläufe junger Zuwanderer beschreibt sie Mechanismen der institutionellen Diskriminierung und beleuchtet Faktoren, die sie verursachen. Sprachkenntnisse von Kindern mit nicht-deutscher Muttersprache werden als eins von mehreren Kriterien dargestellt, die selektierend wirken, wo Kinder nicht der Norm entsprechen (Aydın-Canpolat 2018: 100). Unter Einbeziehung vorheriger Studien (Gomolla \& Radtke 2009, in: Aydın-Canpolat 2018: 278-279) betont die Autorin, dass Sprachdefizite bei Schülern mit Migrationshintergrund „häufig und systematisch" mit einer Lernbehinderung gleichgesetzt zu werden scheinen. Außerdem wird laut Aydın-Canpolat (2018: 102) in deutschen Schulen fehlende Sprachkompetenz der Eltern teilweise auf ihre Kinder projiziert. In Grundschulen werden von den Entscheidungsträgern festgestellte Sprachdefizite häufig als Vorbehalt verwendet, um Kinder mit Migrationshintergrund überdurchschnittlich oft Sonderschulen zuzuweisen. Solche Praktiken, die Aydın-Canpolat (2018) als indirekte Diskriminierung bezeichnet, zeigen einen deutlichen Zusammenhang zwischen den sprachlichen Fähigkeiten von Kindern mit nicht-deutschen Muttersprachen und ihren Chancen auf Erfolg im deutschen Bildungssystem.

\section{Mehrsprachigkeit an der Schule im Kontext deutscher Sprachpolitik}

Der Umgang mit Sprachvielfalt in öffentlicher Bildung steht grundsätzlich in Zusammenhang mit allgemeineren, nationalen Zielsetzungen. In Deutschland unterliegt Sprachpolitik dabei einigen strukturellen und ideologischen Besonderheiten. Generell zeichnet sie sich auf staatlicher Ebene durch ein Fehlen dezidierter Strategien aus. Dieser teils historisch begründete passive 
ENGELS

Ansatz („Tradition des Laisser-faire“, Marten 2016: 145) hinsichtlich sprachpolitischer Maßnahmen und, damit einhergehend, das Fehlen einer übergeordneten Instanz, resultieren in einer breiten Streuung der Verantwortung für den Umgang mit Mehrsprachigkeit im gesellschaftlichen und institutionellen Bereich. Die Entscheidungsgewalt über entsprechende Richtlinien und Maßnahmen liegt daher größtenteils bei einzelnen Verantwortlichen, die sprachpolitische Strategien auf Grundlage ihrer eigenen Erfahrungen und der Bedürfnisse ihres spezifischen Umfeldes entwickeln. Adler (2019: 198) beschreibt im Zusammenhang mit einem Mangel an sprachpolitischer Steuerung ein Spannungsfeld zwischen der Wahrnehmung Deutschlands als einsprachigem Raum und der tatsächlichen Sprachvielfalt. In diesem Spannungsfeld agieren unter anderem die Akteure lokaler Schulpolitiken, die unter dem Eindruck einsprachig orientierter bildungspolitischer Leitlinien stehen und gleichzeitig mit teilweise enormer sprachlicher Vielfalt an ihren Schulen konfrontiert sind.

Im Bereich der Schulpolitik sind Schulen grundsätzlich an die Bildungspläne der jeweiligen Länder gebunden. Wo lokale Änderungen oder Ergänzungen dieser Pläne nötig erscheinen - etwa bei einer hohen Anzahl an Schülern, die Deutsch als Zweit- oder Drittsprache lernen - wird sich oftmals auf den Bereich der Sprachförderung bezogen.

Die Kombination dieser Faktoren - die Eigenverantwortung der Schulen und das Fehlen einer dezidierten Sprachpolitik bzw. der Behandlung sprachpolitischer Fragen als solche - hat im schulischen Bereich zu einer enormen Bandbreite an Sprachförderprojekten und ihren jeweiligen individuellen Ansätzen geführt. Die Entscheidungen der Verantwortlichen in solchen Projekten, und ihr Verständnis von Mehrsprachigkeit in Bildung, hat direkte Auswirkungen auf junge Lernende. Der folgende Teil der hier vorgestellten Arbeit beschäftigt sich daher mit dem Diskurs über Mehrsprachigkeit unter Akteuren der Sprachförderung an Schulen in Nordrhein-Westfalen.

\section{Theoretische Grundlagen der Diskursanalyse}

Der Ansatz der Kritischen Diskursanalyse (KDA) wird zu großen Teilen durch das zugrundeliegende Verständnis von Diskurs und Ideologie geprägt. Da in diesem Bereich eine Vielzahl von Konzepten existiert, sollen hier zunächst die Begrifflichkeiten „Diskurs“ und „Ideologie“ im Sinne der nachfolgenden Analyse diskutiert werden. Konzepte von Diskurs können zwischen akademischen Fachrichtungen variieren und unterschiedliche Aspekte von Diskurs hervorheben. Grundlage für den in dieser Arbeit vorgestellten Analyseansatz ist eine soziolinguistische Perspektive auf Diskurs, die diesen als soziales Handeln versteht. 
Gee (1999) unterscheidet dabei zwischen discourse und Discourse, wobei Ersteres das Gesprochene - z.B. Gespräche an sich - und Letzteres den Gebrauch von Sprache und seine sozial-interaktive Komponente versteht (Gee 1999: 17). Diese Auffassung von Sprache als Handeln, das von sozialen Konventionen bestimmt wird, führt Gee (1999: 18) so weit, dass er Mitglieder einer Gesellschaft als Träger (carrier) eines Diskurses bezeichnet, welcher schon vor dem Sprecher selbst existierte und nach ihm weiter existieren wird. Gee (1999: 89) diskutiert auch den Zusammenhang zwischen Sprache und Realität, den er als Reflexivität (reflexivity) bezeichnet: das Wechselspiel zwischen Realität und Sprache, wobei Sprache Realität verändern kann und umgekehrt. Der Autor hebt damit die der folgenden Analyse zugrundeliegende Auffassung hervor, dass Diskurs eine (soziale) Situation gleichzeitig widerspiegeln und sie verändern kann.

Der Begriff der Ideologie kann im Rahmen dieses Textes nicht erschöpfend erörtert werden. Ganz allgemein kann Ideologie definiert werden als „eine von einem bestimmten Blickwinkel aus organisierte, systematische Anordnung von Vorstellungen“ (Kress und Hodge 1993, in: Mooney und Evans 2015: 6). Wichtig für die nachfolgende Analyse ist, dass diese Vorstellungen, internalisiert und oftmals nicht bewusst gemacht, das Verhalten von Mitgliedern einer Gesellschaft mitbestimmen. Nach Mooney und Evans (2015: 16) stellt jede Entscheidung für eine bestimmte sprachliche Ausdrucksweise eine ideologische Entscheidung dar; deshalb kann die Untersuchung von Sprache helfen, Strukturen und Inhalt von Ideologien sichtbar zu machen.

KDA bezeichnet weniger eine konkrete Methode als eine interdisziplinäre Perspektive auf die Analyse von Sprache, die über die inhaltliche Bedeutung des Gesagten hinaus geht. Da sich KDA auf eine Vielfalt von Ansätzen und die dazugehörigen akademischen Traditionen bezieht, ist eine enge Definition des Begriffs problematisch.

Taylor (2013: 4) beschreibt KDA allgemein als einen „Ansatz der Analyse von Gesprochenem und Text, der Machtmechanismen der Gesellschaft und die Rolle von Sprache bei der Etablierung und der Aufrechterhaltung von Dominanz berücksichtigt"3. Die Annahme, dass Sprache und Macht in der Gesellschaft eng verknüpft sind, stellt eine der wichtigsten Grundlagen von KDA dar. Durch diesen Zusammenhang wird impliziert, dass bestehende Machtverhältnisse und sozialer Wandel sich in Sprache äußern. Einer der analytischen Aspekte in KDA besteht demnach darin, solche Zusammenhänge in Diskursen aufzuzeigen.

3 Originaltext: „[...] an interdisciplinary approach to the analysis of talk and texts which considers the workings of power in society and the role of language in establishing and perpetuating dominance" 
ENGELS

Das kritische Element, das in KDA über die bloße Analyse hinaus geht, besteht darin, solche Zusammenhänge aufzuzeigen, wo sie nicht offensichtlich sind oder nicht bewusst wahrgenommen werden. Laut Fairclough (2001: 229) sollten daher soziale Probleme und Veränderungen Ursprung und Gegenstand von KDA sein. Hier sollte die Frage gestellt werden, welche Veränderungen im Zusammenhang mit sozialen Problemen stattfinden, und wie sich diese Veränderungen in Sprache äußern bzw. durch Sprache verursacht werden. Darüber hinaus geht Fairclough (1995: 27) davon aus, dass dominierende Gruppen in einer Gesellschaft ihre Ideologien durch Diskurs normalisieren. Aufgabe der KDA soll demnach sein, solche Ideologien zu denaturalisieren.

Van Leeuwen (2008: 6) bezeichnet Diskurs, ähnlich wie oben beschrieben, als Teil sozial regulierten Verhaltens. Aufbauend auf diesem Verständnis stellt er ein umfassendes theoretisches Konzept für KDA zur Verfügung, das die Analyse von der durch Sprache ausgeführten sozialen Handlung aus beginnt, soziale Rollenverteilung miteinbezieht und dann erst die linguistischen Eigenschaften des Gesagten untersucht (Van Leeuwen 2008: 23).

Ein wesentlicher Bestandteil von Van Leeuwens (2008) Theorie ist die soziale Praxis der Legitimation (legitimation). In dieser Definition meint Legitimation das Beantworten der verbalisierten oder unausgesprochenen Frage danach, warum etwas auf eine bestimmte Art und Weise getan werden sollte. Van Leeuwen (2008:105) betont, dass diese Art des diskursiven Handelns immer einen großen Teil von Sprache darstellt und demnach oft in Diskursen zu finden ist. Das häufige Vorkommen dieser Praxis selbst muss also nicht grundsätzlich als Versuch der Rechtfertigung interpretiert werden. Die Untersuchung verschiedener Arten von Legitimation kann nach Meinung des Autors allerdings helfen, ideologische Kräfte oder Autoritäten hinter den Äußerungen von Sprechern zu identifizieren.

Unter dem Oberbegriff der Legitimation definiert Van Leeuwen (2008) mehrere Unterklassen, die sich sowohl in ihrer inneren Logik als auch in ihren sprachlichen Charakteristika voneinander unterscheiden. Zwei dieser Unterklassen sind relevant für die Analyse des hier untersuchten Diskurses: die der Moralischen Bewertung (moral evaluation) und die der Rationalisierung (rationalization).

Moralische Bewertungen äußern sich laut Van Leeuwen (2008: 106) größtenteils durch Adjektive, die eine positive oder negative Konnotation tragen. Als offensichtliches Beispiel für solche Adjektive führt er „gut" und „schlecht“ an. An anderen Beispielen, die sowohl beschreibende als auch wertende Eigenschaften haben, wie „natürlich“, „gesund“ und „cool“, zeigt der Autor auf, dass die Konnotation solcher Adjektive von dem Wertesystem abhängt, innerhalb dessen sie geäußert werden. Gleichzeitig wird ein solches 
Wertesystem nicht in jedem Kontext klar benannt. Dementsprechend kann KDA konnotierende Adjektive nicht interpretieren, sondern nur aufzeigen. Van Leeuwen (2008: 110) erklärt außerdem, dass Forschende moralische Bewertungen anhand ihres eigenen kulturellen Wissens erkennen und benennen können. Hier wird die besondere Rolle des Wissenschaftlers deutlich, der in KDA Wertesysteme berücksichtigt, deren Teil er oftmals selbst ist.

Besondere Aufmerksamkeit lässt Van Leeuwen (2008: 111) dem oben genannten Beispiel „natürlich“ zukommen. Während der Begriff seiner Auffassung nach als moralische Bewertung bezeichnet werden kann, ist nicht klar, an welcher Stelle sich hier moralische Ordnung und die Wahrnehmung einer natürlichen Ordnung vermischen bzw. unterscheiden. KDA kann nach Meinung des Autors nicht leisten, diese beiden Bereiche zu unterscheiden, und sieht es auch nicht als ihre Aufgabe an. Auch und besonders hier sollte sich der Analysierende also auf die Benennung des Adjektivs beschränken und von Interpretationen Abstand nehmen.

Eine andere Form der Legitimation, die der Instrumentalen Rationalisierung (instrumental rationalization), zeichnet sich durch eine zweckgebundene Argumentation aus (Van Leeuwen 2008: 113). Sie ist damit abzugrenzen gegen andere Strategien (wie etwa die Berufung auf Autoritäten oder Traditionen), da die Art und Weise, wie etwas getan wird, durch einen bestimmten Zweck, einen instrumentalen Wert, legitimiert wird. Die Identifizierung eines Zwecks und seine Priorisierung können dabei an unterschiedliche Werte gebunden sein.

Die diskursiven Praktiken der Instrumentalen Rationalisierung und der moralischen Bewertung stellen zwei Hauptaspekte der Analyse des untersuchten Diskurses da.

\section{Ergebnisse}

Der untersuchte Diskurs besteht aus fünf teilstrukturierten Interviews mit Experten in Sprachförderung in Nordrhein-Westfalen. Alle Befragten waren zum Zeitpunkt der Datenerhebung aktiv in die Entwicklung von Sprachfördermaßnahmen involviert und bei städtischen, kommunalen oder nordrhein-westfälischen Trägern beschäftigt. Allen war gemeinsam, dass sie sich nach Ausbildung und Tätigkeit als Lehrende an Regelschulen bzw. eines Kindergartens aus eigener Motivation in Sprachförderprojekte eingebracht hatten. Die Interviews wurden mit Zustimmung der Befragten aufgezeichnet und teilweise transkribiert. Die Transkriptionen dienen als Grundlage für die vorliegende Analyse. 
ENGELS

Auffälligstes Merkmal des untersuchten Diskurses war ein genereller Mangel an Bezug zum Konzept individueller Mehrsprachigkeit. Da Mehrsprachigkeit im Zentrum der Fragestellung der vorliegenden Arbeit stand, war der Begriff Teil des in den Interviews verwendeten Fragenkatalogs. Dennoch bezogen sich die Befragten selten auf Mehrsprachigkeit. Vielmehr schienen die untersuchten Äußerungen auf eine Unterscheidung mehrerer verschiedener Konzepte von Sprachen hinzudeuten, wobei Deutsch und nichtdeutsche Sprachen sowohl in ihrer Konzeption als auch in ihrer (qualitativen) Bewertung voneinander getrennt beschrieben wurden. Von drei ausdrücklichen Bezügen auf Mehr- bzw. Zweisprachigkeit gingen zwei mit moralischen Bewertungen nach Definition von Van Leeuwen (2008) einher.

In Bezug auf Sprachkompetenz bei Schülern lassen sich im untersuchten Diskurs Unterschiede in der Qualitätsbewertung, der Zuordnung von Sprachen zu Sprechern und der Gewichtung der Rolle von Deutsch und anderen Sprachen in der Bildung benennen. Bezogen auf die vorhandenen oder gewünschten Deutschkenntnisse von Schülern benutzten die Befragten oft Begriffe der Richtigkeit (radebrechend; korrekt; richtig; nicht richtig; falsch). Im Gegensatz zum Qualitätsmerkmal Korrektheit wurden der Erwerb oder das Beherrschen einer nicht-deutschen Sprache oft im Kontext von Authentizität beschrieben. Bezeichnungen wie vernünftig; solide; echt; wirklich gut, die im Zusammenhang mit dem Spracherwerb von Kindern geäußert wurden, weisen hier auf ein Verständnis von Spracherwerb hin, das einer Form von unechtem, unzureichendem Spracherwerb gegenüber zu stehen scheint. Besonders deutlich wird der Kontrast in der folgenden Äußerung, in der die entsprechenden Ausdrücke in Fettschrift hervorgehoben sind:

(1) Aber bei der Spracherfahrung sind wir inzwischen auf dem Stand, dass wir sagen, wenn ein Kind seine eigentliche Muttersprache gut gelernt hat. Und seine eigene Muttersprache gut spricht. Auf einer soliden Struktur, einer soliden Basis. Dann lernt es auch gut die zweite Sprache. Notfalls auch die dritte. Das ist einfach inzwischen klar. [...] Es ist viel besser, wenn die in ihrer Familie ihre Muttersprache vernünftig lernen. Und das Kind wirklich eine super gute Basis, ein gutes Fundament hat in der eigenen Sprache. Da kriegen wir die deutsche Sprache drauf.

Diese qualitative Unterscheidung von Sprachen - im Zusammenhang mit ihrem Erwerb - voneinander trat darüber hinaus auch in einer klaren $\mathrm{Zu}$ ordnung der Sprachen zu Sprechern zutage. Wie in Beispiel (1) mit Unterstreichungen hervorgehoben ist, wurde mehrsprachigen Kindern oft der Besitz von nicht-deutschen Sprachen zugesprochen, für Deutsch dagegen nie. Neben dem Gebrauch von Possessivpronomen äußerte sich diese Zuordnung 
in begleitenden Adjektiven. Einige Beispiele für die Zuordnung sind die Formulierungen Halten Sie Ihre Sprache hoch, sprechen Sie Ihre Sprache; die Sprache der Kinder; ihre eigene Sprache.

Auch das folgende Beispiel, in dem nicht-deutsche Sprachen ihren Sprechern zugeordnet werden, in dem solche Formulierungen aber für die deutsche Sprache fehlen, illustriert diese Distanzierung deutlich:

(2) Ich halte diesen herkunftssprachlichen Unterricht auch für wichtig, dass die in ihrer Muttersprache ein gutes Niveau erreichen. [...] Weil - je größer mein Wortschatz in meiner Muttersprache, desto einfacher fällt es mir, diesen Wortschatz auch im Deutschen zu erreichen. Aber: für die Zukunft, wenn ich eine Lehre anfangen will, brauche ich Deutsch. Das ist einfach so. Wenn ich studieren will - in Deutschland - dann brauche ich die deutsche Sprache.

Einer der Befragten äußerte sich zu diesem Thema auf andere Weise. Im Kontext einer sprachpolitischen Forderung leitete er von linguistischer Diversität in Deutschland eine Verantwortung des Staates für muttersprachlichen Unterricht $\mathrm{ab}$, indem er alle von Kindern gesprochene Sprachen der deutschen Gesellschaft zuordnete:

(3) $[\ldots]$ und ich finde es auch nicht richtig, weil das unsere Kinder sind, das sind unsere Sprachen auch, und deswegen ist es staatliche Aufgabe. Deswegen in Nordrhein-Westfalen auch mutter- oder herkunftssprachlicher Unterricht.

In Beispiel (2) findet sich neben einem Ausdruck von Besitz von Sprachen ein weiteres Merkmal des untersuchten Diskurses: Die letzten drei Sätze drücken eine Priorisierung von Deutsch als Bildungssprache aus und ordnen ihr einen instrumentellen Wert zu. In fast allen Interviews wurde der Erwerb von Deutsch als Bildungs- und Landessprache explizit dem (formalen) Erwerb nicht-deutscher Sprachen übergeordnet. Ein Großteil der damit verbundenen Äußerungen nutzte das Argument von Erfolg im Bildungssystem selbst, welcher nur mit umfassenden Kompetenzen in der deutschen Sprache zu erreichen sei, wie in weil man eine Berufsausbildung braucht; weil man eine höhere Schule besuchen muss; es läuft alles über Sprache ab. Einige Befragte bezogen sich auf Zeitpunkte in der späteren Karriere, wie in die Sprache, mit der man auch später 'ne Chance hat, tatsächlich in sein Leben zu starten. Während der Erwerb nicht-deutscher Sprachen von allen Befragten als wichtig bezeichnet wurde, wurde diesen Sprachen an keiner Stelle ein instrumenteller Wert zugeordnet, welcher über die Funktion als Grundlage für das Erlernen des Deutschen hinaus ginge. Dieses Muster lässt sich als diskursive Praktik der instrumentellen Rationalisierung nach Van Leeuwen (2008) bezeichnen, im Zuge derer 
ENGELS

durch Zuordnung eines praktischen Zwecks die bestehende (und gewünschte) Überordnung von Deutsch über andere Sprachen in Bildung legitimiert wird.

Der Begriff der Mehrsprachigkeit bzw. Zweisprachigkeit kam in zwei Fällen explizit vor: in der Äußerung von „guter Zweisprachigkeit“ und „natürlicher Mehrsprachigkeit“. Beide Begriffe können als moralische Bewertungen nach Van Leeuwen (2008) bezeichnet werden.

Im folgenden Beispiel begründet einer der Befragten seine Handlungsempfehlung für Eltern zweisprachiger Kinder:

(4) Wenn Papa eher nur Arabisch spricht und nicht gut Deutsch, dass das Kind mit Papa dann grundsätzlich nur Arabisch spricht, dass Sie als gut Deutsch sprechende Person mit dem Kind nur Deutsch sprechen, und dass dadurch eine echte, wirklich gute Zweisprachigkeit dann eben auch erzeugt wird. (Was ist gute Zweisprachigkeit für Sie?) Gute Zweisprachigkeit ist natürlich, wenn das Kind beide Sprachen gut beherrscht. Und zwar bis auf ein Bildungssprachenniveau gut beherrscht.

In diesem Beispiel scheint das wie oben beschriebene Verständnis von Echtheit im Zusammenhang mit Spracherwerb anzuknüpfen. Im Unterschied zu einer sonst häufig zu beobachtenden konzeptionellen Trennung von Sprachen scheinen in dieser Äußerung aber die Faktoren der Qualität und der Echtheit sowohl auf Deutsch als auch auf nicht-deutsche Sprachen bezogen zu werden - aus den Konzepten Authentizität und Korrektheit wird hier echt, wirklich gut.

Den Begriff der natürlichen Mehrsprachigkeit erklärte ein anderer Befragter auf direkte Nachfrage als ein Konzept, das Mehrsprachigkeit als ein festes Merkmal von Migranten versteht, als eine wichtige Kompetenz, die die Kinder entfalten müssen, um nicht zwischen den Stühlen zu sitzen und die nicht per Instruktion erworben wird. Seiner Auffassung nach signalisiert die Formulierung eine Perspektive auf Mehrsprachigkeit, die diese nicht als Problem begreift.

Bemerkenswert ist hier der Gebrauch des Adjektivs „natürlich“, welches Van Leeuwen (2008) als problematisch in der Diskursanalyse hervorhebt. Obwohl der Ausdruck nur von einem Befragten geäußert wurde, kommt ihm im Kontext der Sprachförderung besondere Bedeutung zu: natürliche Mehrsprachigkeit ist im sogenannten Teilhabegesetz des Landes NordrheinWestfalen verankert, welches unter anderem die Grundlage für staatliche finanzierte Sprachfördermaßnahmen darstellt. ${ }^{4}$

4 Vgl. Gesetz zur Förderung der gesellschaftlichen Teilhabe und Integration in NordrheinWestfalen, $\$ 2$ (3). 
Von der diskursiven Praktik der moralischen Bewertung abzugrenzen ist das nächste Beispiel, welches allerdings ebenfalls eine Form von Konzeption von Mehrsprachigkeit darstellt.

Dem Verständnis von einer Art authentischer, vollständiger Mehrsprachigkeit, wie in Beispiel (4) beschrieben, steht in der Äußerung einer anderen Befragten der Begriff der „Halbsprachigkeit“ gegenüber:

(5) Und dann verkümmert die Muttersprache, und das Deutsche wird auch nicht richtig gelernt, und dann habe ich diese, wie es so schön heißt, diese Halbsprachigkeit. Und dann lieber die Muttersprache richtig lernen und... wenn ich einen komplexen Satzbau schon habe oder auch Wortschatz. Dann fällt es mir leichter, eine andere Sprache auf einem besseren Niveau zu lernen, als wenn ich - ja, gar keine Sprache hab.

\section{Diskussion}

Die Datengrundlage für den vorliegenden Text stellt lediglich eine Stichprobe im nordrhein-westfälischen Diskurs um Mehrsprachigkeit dar. Während die Ergebnisse daher keine Verallgemeinerungen zulassen, und Spekulationen über die Erfahrungen und Gedanken der Befragten - ganz besonders in Kritischer Diskursanalyse - unzulässig sind, lassen sich die oben beschriebenen Schwerpunkte dennoch mit vorangegangener Forschung verknüpfen. Im Folgenden möchte ich auf einige Beobachtungen in der eingangs beschriebenen Literatur eingehen und erläutern, wie sich Erkenntnisse anderer Autoren im hier behandelten Diskurs widerspiegeln. Anschließend gebe ich einen Ausblick auf potentielle zukünftige Anknüpfungspunkte in der Diskursanalyse und mögliche Implikationen der derzeitigen Situation für gegenwärtige und zukünftige Bildung von mehrsprachigen Kindern.

Einen wesentlichen Schwerpunkt in der vorliegenden Analyse stellt die Tatsache dar, dass das ursprünglich zu erforschende Konzept Mehrsprachigkeit unter den Befragten keine vordergründige Rolle zu spielen scheint. Das Wort Mehrsprachigkeit wurde in den Interviews nur selten und in zwei Fällen in Verbindung mit moralischen Bewertungen verwendet. Wie eingangs erwähnt, deckt sich ein augenscheinlicher Mangel an Bezug zu soziolinguistischen Themen wie der Mehrsprachigkeit mit früheren Forschungsergebnissen von Thomauske (2011) und lässt sich einem allgemein geringen Bewusstsein für die mehrsprachige Entwicklung deutscher Kinder sowie für andere sprachplanerische und -politische Themen zuordnen. Allerdings muss festgehalten werden, dass das Thema Mehrsprachigkeit, wenn von Seiten der Befragten auch nicht explizit aus sprachwissenschaftlicher Perspektive definiert, omnipräsent 
ENGELS

in den hier analysierten Interviews war. Alle Informanten waren Experten für Sprachförderung und von ihren Arbeitgebern zur Weiterbildung angehalten. Jede/r von ihnen äußerte klare Vorstellungen davon, wie am Besten mit dem Spracherwerb des Deutschen und anderer Sprachen in Bildung umzugehen sei und berief sich dabei auf (vermeintlich) wissenschaftliche Erkenntnisse oder sprachpolitische Strategien. Das bloße Fehlen des Wortes Mehrsprachigkeit an sich, auf das die Fragestellung dieser Arbeit explizit abzielte, beweist also keinen Mangel an Bewusstsein für die Problemstellungen, mit denen die Befragten, wie oben beschrieben, in ihrer Arbeit selbst konfrontiert waren was es allerdings zu betonen scheint, ist ein Mangel an Bewusstsein für sprachwissenschaftliche Erkenntnisse.

Der Unterschied zwischen wissenschaftlicher und nicht-wissenschaftlicher Grundlage für Konzepte von Mehrsprachigkeit lässt sich anhand der Begriffe „natürliche Mehrsprachigkeit“ und „gute Zweisprachigkeit“ verdeutlichen. Beide Bezeichnungen stellen aus diskursanalytischer Sicht moralische Bewertungen dar und entbehren in dieser Formulierung einer wissenschaftlichen Grundlage. Auch das Konzept der „Halbsprachigkeit“, auf das sich eine Befragte in Beispiel (5) bezog, stellt einen Mythos dar (vgl. Wiese 2011). Die Wahrnehmung eines im Prozess des mehrsprachigen Spracherwerbs befindlichen Kindes als inkompetent, weil es eine Sprache nicht richtig beherrscht, oder weil eine Sprache verkümmert, wird ebenfalls von Thomauske (2017) besprochen und von ihr als „Tradition des sprachlichen Handicaps“ in den Kontext von nationalistischer Sprachideologie der vergangenen Jahrhunderte eingeordnet. Am Begriff der „Halbsprachigkeit“ wird die von Thomauske (2017) und Aydın-Canpolat (2018) beschriebene Problematisierung von Phänomenen des mehrsprachigen Spracherwerbs besonders deutlich. Auf ähnliche Art und Weise lässt das in Beispiel (5) geäußerte Verständnis von „gar keine Sprache haben“ mit der Wahrnehmung von Sprachlosigkeit bei Kindern verknüpfen, auf die Thomauske (2017) im Titel ihrer Dissertation anspielt.

An die Frage, wer (welche) Sprache hat, knüpft ein weiterer Schwerpunkt der Analyse an: für die qualitative Unterscheidung zwischen Deutsch und anderen Sprachen war unter anderem charakteristisch, dass mehrsprachigen Kindern oftmals der Besitz „ihrer“ Muttersprachen zugesprochen wurde, der von Deutsch dagegen nie. Gleichzeitig wurden im Zuge von rationellen Instrumentalisierungen dem Erwerb der deutschen Sprache häufig praktische Zwecke zugeordnet, für nicht-deutsche Sprachen dagegen äußert selten. Dies stimmt überein mit der eingangs beschriebenen Hierarchisierung von Sprachen in Bildung, oder genauer: einer empfundenen Notwendigkeit für Deutsch als einziger Bildungssprache in Deutschland, für die instrumentelle Rationalisierungen als diskursive Legitimation dienten. 
Die hier analysierten diskursiven Praktiken ergeben also ein Bild, in dem Kindern der Besitz nicht-deutscher Sprachen zugeordnet wird - gleichzeitig wird nur der deutschen Sprache ein Zweck im Bildungsweg derselben Kinder zugesprochen. Hier könnte eine Verbindung zwischen dem untersuchten Diskurs und dem eingangs erwähnten allgemeinen Verständnis von Deutschland als letztlich einsprachigem Nationalstaat bestehen. Damit in Zusammengang können auch die Unterschiede in der Qualitätsbewertung von Sprachkompetenz bei jungen Lernenden gesehen werden: Objektiv bewertbare - also als „richtig“ oder "falsch“ deklarierbare - Aspekte von Sprache waren im untersuchten Diskurs für Deutsch prävalent, für nicht-deutsche Sprachen schien dagegen eine Bevorzugung von Authentizität vorzuherrschen. Betrachtet man formale Aspekte wie Grammatik und Rechtschreibung einer Sprache, so ergibt sich aus diesem Verständnis von Sprachen und ihrer Rolle eine Notwendigkeit in Bildung, formale Aspekte des Deutschen zu lehren, nicht jedoch für andere Sprachen.

In diesem Verständnis, hier aus vorhergehender Literatur und der vorliegenden Analyse zusammengesetzt, trägt Schule in Deutschland die Verantwortung für die Vermittlung des Deutschen, nicht jedoch für die Ausbildung von Multiliterarität bei Kindern, die bis zu ihrem Eintritt in die Grundschule mit einer nicht-deutschen oder mehreren Sprachen aufgewachsen sind. Während repräsentative Daten bislang rar sind, lässt sich vermuten, dass dieser Prozess des Wechsels von mehrsprachigem Alltag zu einsprachig deutscher Bildung bis zu einem Drittel der jungen Lernenden in Deutschland betreffen könnte. Einige negative Konsequenzen eines solchen einsprachig orientierten Ansatzes in der Schule sind im Verlauf dieses Textes behandelt worden - mangelndes Wissen um die Entwicklung mehrsprachiger Kinder kann, wie von Aydın-Canpolat (2018) erörtert, zu Diskriminierung beitragen oder gar zum Verstummen von Kindern führen, welche den Normen ihrer Lernumgebung zunächst nicht zu entsprechen vermögen.

Einer Problematisierung dieser Situation könnte ein Ansatz gegenüberstehen, der de facto vorhandene Sprachvielfalt als Realität anerkennt, wo sie vorhanden ist, eine vielsprachige Schülerschaft als Potential begreift und ermöglicht, die individuelle Entwicklung einzelner Kinder zu fördern. $\mathrm{Zu}$ berücksichtigende Faktoren eines solchen Ansatzes wären nicht nur Veränderungen in der Schulpolitik und dem Umgang mit Mehrsprachigkeit in der Schulgemeinschaft, sondern auch und vor allem im Deutschunterricht, der im traditionellen Sinne Deutsch als Muttersprache voraussetzt und mittlerweile an einen erheblichen Teil von Schülern gerichtet ist, die Deutsch als Zweit- oder Drittsprache lernen. Wie Gerlind Belke (2016: 28) in ihrem Buch Mehr Sprache(n) für alle ausführlich diskutiert, könnte die Zusammenführung 
von Mutter- und Fremdsprachendidaktik - unter Zuhilfenahme mehrsprachiger Literatur und Poesie - dabei helfen, Deutschunterricht so zu konzipieren, dass er für alle Schüler zugänglich und sinnvoll ist. Ein solcher Unterricht, der insbesondere den Schriftspracherwerb und literarisches Lernen als Grundlage für den weiteren Bildungsweg hervorhebt, käme laut Belke (2016: 29) allen Kindern zugute. Bedingung für einen derartigen Wandel wären wohl das weitverbreitete Verständnis und die gesellschaftliche Akzeptanz von sprachlicher Diversität als Regelfall an vielen deutschen Schulen, sowie der Anspruch, diese Diversität als Ausgangspunkt für die Weiterentwicklung bildungspolitischer Strategien oder Didaktiken, anstatt als Abweichung von einer als einsprachig angenommenen Normalität anzusehen. Im untersuchten Diskurs finden sich auch Legitimationen für diese Ansicht, wie am Beispiel der „natürlichen Mehrsprachigkeit" und in Beispiel (3) hervorgehoben. Dieser Teil des hier behandelten Diskurses und die Tatsache, dass „natürliche Mehrsprachigkeit“ in nordrhein-westfälischer Gesetzgebung verankert ist, könnten Hinweise auf die soziale Veränderung sein, die Fairclough (2001) als Gegenstand kritischer Diskursanalyse vorschlägt.

\section{Ausblick}

Der vorliegende Text analysiert einen sehr begrenzten Ausschnitt aus dem Diskurs um Mehrsprachigkeit unter Experten für Sprachförderung in NordrheinWestfalen. In den Kontext von bisheriger sprachpolitischer Situation, vorhergehender Literatur und diskursanalytischen Ansatz eingeordnet ergibt sich ein Bild, das zwar in Teilen den Erkenntnissen anderer Autoren entspricht; allerdings finden sich im recht diversen Diskurs fünf unterschiedlicher Befragter durchaus differenzierte und teilweise gegensätzliche Auffassungen, die jeweils durch diskursive Praktiken der Legitimation ausgedrückt wurden. Der Bereich der Sprachpolitik ist in Deutschland bisher recht wenig erforscht, genauso wie die tatsächliche Anzahl von Sprachen und mehrsprachigen Kindern an deutschen Schulen. Der Teilbereich Sprachförderung, der linguistische Realität und die Anforderungen einer einsprachig orientierten Bildungspolitik zu vereinen versucht, kann als sehr divers und dynamisch bezeichnet werden. Gerade innerhalb dieser Sphäre von oftmals nicht-benannten sprachpolitischen Aushandlungen kann kritische Diskursanalyse helfen, Einblicke in den aktuellen Diskurs und potentiell darin ablesbare Prozesse der Veränderung zu gewinnen. Ausgehend vom Fokus der vorliegenden Arbeit könnte dabei beispielsweise eine kritische Analyse bestimmter im Integrations-, Bildungs- und Mehrsprachigkeitsdiskurs vorherrschender Begriffe unter Einbeziehung ihrer historischen Entwicklung sinnvoll sein. Einen anderen Ansatzpunkt könnte die 
systematische Analyse eines größeren Ausschnitts von Diskurs unter jeweils als homogen angenommenen Gruppen wie Lehrern, Eltern oder Kindern darstellen. Auch eine Untersuchung des öffentlichen (schriftlichen) Diskurses über Mehrsprachigkeit könnte zu einem besseren Verständnis der derzeitigen Situation und eventuell stattfindender Veränderungen beitragen. Letztlich ist in einem Land ohne dezidierte Sprachpolitik und mit einem womöglichen Mangel an Kommunikation von Fachwissen entscheidend, wie Sprache verwendet wird, um über Sprache(n) zu sprechen; und um sich hierfür Sprache bedienen zu können, bedarf es der Ausbildung von (Multi-)Literarität aller Kinder im deutschen Bildungssystem.

\author{
Farin Engels \\ farin_engels@web.de \\ University of Marburg / University of Tartu \\ GERMANY / ESTONIA
}

\title{
Literaturverzeichnis
}

Adler, A. 2019. Sprachstatistik in Deutschland. - Deutsche Sprache, 3.19, 197-219. https://doi.org/10.37307/j.1868-775X.2019.03.02

Artelt, C., Baumert, J., Klieme, E., Neubrand, M., Prenzel, M., Schiefele, U., Schneider, W., Schümer, G., Tillmann, K.-J. und M. Weiß, Hg. 2001. PISA 2000. Zusammenfassung zentraler Befunde. Berlin: Max-Planck-Institut für Bildungsforschung. https://doi.org/10.1007/978-3-322-83412-6

Autorengruppe Bildungsberichterstattung, Hg. 2016. Bildung in Deutschland 2016. Ein indikatorengestützter Bericht mit einer Analyse zu Bildung und Migration. Bielefeld: Bertelsmann.

Autorengruppe Bildungsberichterstattung, Hg. 2018. Bildung in Deutschland 2018. Ein indikatorengestützter Bericht mit einer Analyse zu Wirkungen und Erträgen von Bildung. Bielefeld: wbv Publikation.

Aydın-Canpolat, G. 2018. Erfolg und Scheitern im Deutschen Bildungswesen. Determinanten der Bildungsverläufe junger Zuwanderer. Dissertation Universität DuisburgEssen, Campus Essen. Wiesbaden: Springer VS.

Belke, G. 2016. Mehr Sprache(n) für alle. Sprachunterricht in einer vielsprachigen Gesellschaft. Baltmannsweiler: Schneider Verlag Hohengehren.

Bundesamt für Migration und Flüchtlinge. 2016. Das Bundesamt in Zahlen 2015. Asyl, Migration und Integration. - http://www.wir-sind-bund.de/SharedDocs/ Anlagen/DE_nvam/Publikationen/Broschueren/bundesamt-in-zahlen-2015. pdf?_blob=publicationFile (18.10.2020).

Fairclough, N. 1995. Critical Discourse Analysis. The Critical Study of Language. London: Longman. 


\section{ENGELS}

Fairclough, N. 2001. The Discourse of New Labour: Critical Discourse Analysis. M. Wetherell, S. Taylor, S. J. Yates, Hrsg., Discourse as Data. A Guide for Analysis. London and Milton Keynes: Sage Publications and The Open University, 229266.

Gee, J. 1999. An Introduction to Discourse Analysis: Theory and Method. London: Routledge.

Hunn, K. 2005. „Nächstes Jahr kehren wir zurück.... Die Geschichte der türkischen "Gastarbeiter" in der Bundesrepublik. Göttingen: Wallstein Verlag.

Konsortium Bildungsberichterstattung, Hg., 2006. Bildung in Deutschland. Ein indikatorengestützter Bericht mit einer Analyse zu Bildung und Migration. Bielefeld: Bertelsmann.

Kress, R., Hodge, G. 1993. Language as Ideology. 2. Auflage. London: Routledge.

Marten, H. 2016. Sprach(en)politik. Eine Einführung. Tübingen: Narr Francke Attempto Verlag.

Mooney, A., Evans, B. 2015. Language, Society and Power. An Introduction. 4. Auflage. London: Routledge.

Stanat, P., Artelt, C., Baumert, J., Klieme, E., Neubrand, M., Prenzel, M., Schiefele, U., Schneider, W., Schümer, G., Tillmann, K.-J. und M. Weiß. 2002. PISA 2000: Die Studie im Überblick. Grundlagen, Methoden und Ergebnisse. Berlin: Max-PlanckInstitut für Bildungsforschung.

Taylor, S. 2013. What is Discourse Analysis? London: Bloomsbury Academic.

Thomauske, N. 2011. The Relevance of Multilingualism for Teachers and Immigrant Parents in Early Childhood Education and Care in Germany and in France. Intercultural Education, 22, 327-336. https://doi.org/10.1080/14675986.2011.61 7425

Thomauske, N. 2017. Sprachlos gemacht in KiTa und Familie. Ein deutschfranzösischer Vergleich von Sprachpolitiken und -praktiken. Wiesbaden: Springer VS.

Van Leeuwen, T. 2008. Discourse and Practice. New Tools for Critical Discourse Analysis. New York: Oxford University Press.

Wiese, H. 2011. Führt Mehrsprachigkeit zum Sprachverfall? Populäre Mythen vom „gebrochenen Deutsch“ bis zur „doppelten Halbsprachigkeit“ türkischstämmiger Jugendlicher in Deutschland. - Ş. Ozil, M. Hoffmann \& Yasemin DayığluYücel, Hrsg., Türkisch-deutscher Kulturkontakt und Kulturtransfer. Kontroversen und Lernprozesse. Göttingen: V\&R unipress, 73-84. 